\title{
Generating Scale-free Networks with Adjustable Clustering Coefficient Via Random Walks
}

\author{
Carlos Herrera and Pedro J. Zufiria \\ Depto. Matemática Aplicada a las Tecnologías de la Información \\ ETSI Telecomunicación, Universidad Politécnica de Madrid \\ Ciudad Universitaria s/n, 28040 Madrid, Spain \\ Email: carlos@hyague.es, pedro.zufiria@upm.es
}

\begin{abstract}
This paper presents an algorithm for generating scale-free networks with adjustable clustering coefficient. The algorithm is based on a random walk procedure combined with a triangle generation scheme which takes into account genetic factors; this way, preferential attachment and clustering control are implemented using only local information. Simulations are presented which support the validity of the scheme, characterizing its tuning capabilities.
\end{abstract}

\section{INTRODUCTION}

Network modelling has become a very active research field after the discovery that many different complex systems share some essential common features which can be gathered in a network model [7]. Although network nodes and links can represent very different entities depending on the phenomenon being analyzed, still some common characteristics seem to be ubiquitous in many models. For instance, common patterns usually appear in social networks ([3], [24], [20]), biology networks ([35], [14]), technological networks ([11], [5]) or information networks ([2], [25]) Many of these features are non-trivial so that the traditional Erdös-Rènyi (ER) model [9] for random graphs is not sufficient to explain the behavior of these systems.

The first feature appearing in real networks which was not gathered by the ER model was the small world effect, defined by two factors: slow increase of network diameter with network growth and the existence of a unexpectedly high number of triangles in the network (clustering). In order to mimic these properties, Watts and Strogatz proposed a new model in [34]. Nevertheless, this model could not represent an additional property also found in many real networks: the distribution of the number of neighbors (degree distribution) follows a powerlaw, which is very different from the distributions predicted by early models (e.g., exponential distribution in the ER model).

In [2] the Barabási-Albert (BA) network generation model was presented where network growing nature and preferential attachment were proved to be two essential features for obtaining scale-free networks which follow a power-law in the degree distribution. The preferential attachment stands for the fact that new vertices added to the network are attached preferentially to high-degree vertices. In case that the

\footnotetext{
${ }^{1}$ This classification of networks according to its nature was proposed by Newman in [22] where a larger number of references for each type of network can be found.
}

preference is linear, the probability to get connected to a given vertex is proportional to its degree. The BA model implements this preferential attachment using global network information to compute such probability:

$$
p_{i}=\frac{k_{i}}{\sum_{j=1}^{n} k_{j}}, n=\text { total number of nodes. }
$$

The existence of a scale-free structure in many real networks has motivated the appearance of a number of new network models trying to reproduce at least one of the already mentioned three main characteristics of real networks (clustering, long tail degree distribution, short diameter ${ }^{2}$. Different approaches have been used: some models are based on a static network size [23], [6], while others work on growing networks [2], [13], [33].

In general, it is expected that the process of adding a new vertex in real world networks would not require the availability of such global information. Along this line, several authors have studied alternative local schemes (employing rules that only involve a vertex and its neighbors) to generate scale-free networks without the use of global parameters [19], [29], [33], [18], [17], [8], [15].

Among them, the use of random-walkers to select node attachment in a network-growth algorithm has been suggested in [1] and successfully employed in [32], [27], [10]. In general, the use of the proposed schemes has been justified on the assumption that a random walk of arbitrary length $l$ will end up on a vertex $i$ of degree $k_{i}$ with probability given in equation (11), i.e., random walks are assumed to generate a pure preferential selection procedure (to be used as the basis of a preferential attachment scheme). The analytical characterization of these random walk models has been performed under some mean-field hypotheses, so that preferential attachment is studied but no other network features are considered. In [33] the correlation between clustering and degree is analysed also under the mean-field hypotheses, but the tuning of the clustering coefficient is not addressed.

In this paper, alternative random walk selection schemes are presented which allow for the control of both preferential

\footnotetext{
${ }^{2}$ Alternatively, some models try to mimic other network properties. For example, the goal of the model presented in [16] is to ensure that the network shows an arbitrary subgraph distribution. In [31], the property to be reproduced is the existence of communities like the ones observed in real social networks; this could be considered as a generalization of clustering control.
} 
attachment and clustering coefficient in the process of growing a scale-free network. The schemes depend on the transition probability distribution of the random walk in a manner that each path sample may have a different size due to a genetic factor. It is shown that the appropriate selection of the mentioned transition probability distribution allows for the tuning of the clustering coefficient of the generated network.

The paper is organized as follows. Section III starts presenting a motivation and the goals of the proposed model for network generation; then such model is described in detail. In Section III simulation results supporting the validity of the model are presented. Finally, Concluding Remarks and Future Work lines are summarized in Section IV

\section{MODEL}

\section{A. Motivation and goals}

Many real world networks are very complex systems governed by several fundamental characteristics. So far, existing network models can only gather some of these features, which may or not be sufficient for the aim of the analysis. Hence, the construction of new more elaborated models addressing the emergence and behavior of additional characteristics is a relevant challenge. As mentioned in the Introduction, one big step in terms of explaining complex networks was the BA Model, where the emergence of a power-law in the degree distribution was explained via two simple assumptions: growing and preferential attachment (PA). The BA model has been a fundamental reference although it presents some limitations.

On the one hand, several authors [10], [27] have pointed out the difficulties of a practical implementation of preferential attachment policies: as defined in the BA model, when a new node is about to join the network, it requires to know the degree of all nodes in the whole network in order to calculate the probability of linking to each existing node. This scheme does not seem to successfully explain the behavior of real-life stages, such as a blogger linking to a web page or a person making new friends (obviously, they do not have or do not employ global network structure information). A new model based only on local schemes was presented in [27], suggesting that PA can be obtained from a random walk (at least in an approximate manner). In [10] this random walk based model is generalized so that the degree distribution can have an exponent different from $\gamma=3$ if a certain fraction $p_{v}$ of edges is created purely at random (i.e, the new node is linked with a randomly chosen existing node, without implementing a walk).

On the other hand, there is also one important feature which cannot be taken into account when employing the original BA model. Although this model performs better than Erdös-Rényi (ER) model concerning the degree distribution, it cannot produce the high clustering coefficient which has been observed in many real networks $3^{3}$ (see table I] presenting results from [21] for online social networks). The clustering coefficient of real networks is known to be higher than the one

\footnotetext{
${ }^{3}$ Although there are no analytical results for the clustering coefficient in the BA model, it is known ([1], [22]) that it decays with network size $C \sim$ $N^{-0.75}$ while in real networks $C$ is independent from $N$.
}

Table I

CLUSTERING COEFFICIENT IN REAL NETWORKS, AND IN MODELS OF THE NETWORKS OF THE SAME SIZE

\begin{tabular}{|c|c|c|c|}
\hline Network & $C$ & $C / C_{E R}$ & $C / C_{B A}$ \\
\hline \hline Flickr & 0.313 & 47.2 & 25.2 \\
\hline LiveJournal & 0.330 & 119.0 & 17.8 \\
\hline Orkut & 0.171 & 7.24 & 5.27 \\
\hline Youtube & 0.136 & 36.9 & 69.4 \\
\hline
\end{tabular}

provided by a purely random model, and its value depends on the nature of the network. It usually takes high values for social networks (for example $C=0.79$ for imdb actor network [34]) but there are some networks which exhibit a power-law with a much lower clustering level $(C=0.011$ for a P2P network [26]). In [13] a mechanism for triangle formation was proposed which allowed the control of the clustering coefficient; such mechanism is furtherly developed and employed in this paper.

In [12] some characteristics in the social networks which are related to genetic factors are presented. Concretely, it is shown that the clustering coefficient is one of those heritable network metrics. In fact, there are people who are very likely to introduce friends to any other friends, whereas some other people prefer to keep their friends apart from each other.

In this paper, a new network growing scheme is presented where a triangle formation scheme is furtherly developed to include a genetic factor as the basis for a clustering control mechanism. This genetic factor in the nodes (known to happen in real networks) combines in a very adequate manner with a random walk based node selection procedure, so that only local information is employed in the whole edge addition process.

\section{B. Model description}

As mentioned before, the main aim of the model proposed in this paper is to generate scale-free networks whose clustering coefficient can be controlled by using only local information. The scale-free network is generated via a growing scheme which employs random walks as a local approximation to the preferential attachment criterion. The model proposed here is grounded on a modification of the model presented by Evans and Saramäki (ES model) [27]. The ES model is defined as follows:

- Initial condition: start with a network of $n_{0}$ vertices.

- Growth: each time step a vertex and $m$ edges are added to the network. Note that $m \leq n_{0}$.

- Linking by a random walk (RW): the new vertex $v_{\text {new }}$ is joined with $m$ existing vertices which are selected the following way: a random existing vertex $v_{s}$ is chosen, then a $l$-step random walk starting from $v_{s}$ is performed; the arrival vertex $v_{e}$ obtained at the end of the walk is linked to $v_{\text {new }}$.

Our model makes use of some random walk properties which happen to be very useful to control the appearance of triangles in the network. First of all, let us consider $v_{\text {new }}$ has been linked to a first selected network node $v_{s}$; obviously this link does not generate any triangle. Now it can be seen that a $l=1$ walk starting from $v_{s}$ will provide a new node that, if also linked to $v_{n e w}$, will generate a triangle. This way, selecting nodes 

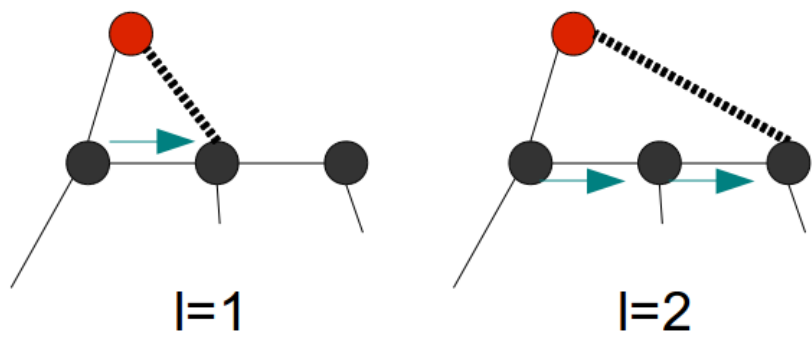

Figure 1. The clustering coefficient is controlled by the following mechanism: if a $l=1$ walk is carried out from the last joined vertex $v_{s}$, we ensure that a triangle is formed. However, if $l=2$ we only generate a triangle if $v_{s}$ and $v_{e}$ are already connected.

via successive $l=1$ walks would add $m-1$ triangles to the network. This fact suggests the possibility of implementing a triangle generation control scheme based on selecting new starting random points for the next walk (avoiding triangle formation), proportionally combined with successive $l=1$ walks (forcing triangle formation). This approach was the first to be analysed: such control mechanism does not behave accurately when reproducing low levels of clustering coefficient. In fact, for a given clustering control parameter, a significant variance was observed, violating our design principle of fine clustering coefficient control (numerical results of this behavior will be showed in section III).

Alternatively, random walks with $l=2$ were employed. Note that if $l=2$ is chosen almost no triangle will be added to the network. In fact, if the seed network with $n_{0}$ vertices does not have any triad, it is straightforward to demonstrate that no triangle will be added (the probability of $v_{s}$ and $v_{e}$ being already connected will be zero). The implications of using $l=1$ or $l=2$ are illustrated in Figure 1

Therefore, controlling the number of $l=1$ and $l=2$ random walks allows for an accurate tuning of the number of triangles added to the network. This might be implemented by just assigning $p_{1}$ probability to $l=1$ walks and $1-p_{1}$ for the $l=2$ case. However, we propose to include this control in a node attribute which is assigned at node's "birth", inspired by the already mentioned results in [12]. In our proposal, any vertex $p$ is assigned a probability $p\left(v_{i}\right)$ which reflects the genetic factor mentioned there upon a certain distribution $f(p)$. This probability remains constant during vertex life and will determine the length of the random walks starting from such node (i.e., a 1-step walk happens with probability $p\left(v_{s}\right)$, being $v_{s}$ the last linked vertex; a 2-step walk is selected otherwise).

Following the explained principles our algorithm is defined as follows:

1) Start with a network of $n_{0}$ vertices. Each vertex is assigned an attribute $p\left(v_{i}\right), i=1 \ldots n_{0}$ according to the random distribution $f(p)$.

2) A vertex $v_{s}$ is chosen randomly.

3) A random walk $l>1$ is performed from $v_{s}$, randomly choosing at each step a neighbor of the current vertex. The arrival vertex $v_{e}$ is marked.

4) Start a new walk from the last marked vertex $v_{l}$. With probability $p\left(v_{l}\right)$ this will be 1-step walk; otherwise will be a 2-step walk. Mark the arrival vertex.

5) Repeat step $4 m-1$ times. Note that $m \leq n_{0}$.

6) Add one vertex to the network. Add $m$ links between the new vertex and the $m$ marked nodes. Assign to the new vertex a probability $p\left(v_{i}\right)$ according to $f(p){ }^{4}$

7) Repeat steps 2 to 6 ( $n-n_{0}$ times).

Hence, the algorithm has the following design parameters: number of nodes $n$, number edges to be attached per vertex $m$, and a probability distribution $f(p)$. Note that, as it is usual in growing network models, $m$ allows to control the average degree since $\langle k\rangle=2 m$. Concerning the distribution $f(p)$, in order to simplify the interpretation of results, a binomial distribution has been chosen to illustrate the scheme in this paper; so there is a fraction $c c$ of nodes with $p\left(v_{i}\right)=1$ having the rest of the nodes $p\left(v_{i}\right)=0$. It is expected that different distributions may lead to different community structures of the resulting networks.

Another interesting issue is the selection of $l>1$ for the first walk, since it is different from previous random walk based models. Although in [27] is stated (and supported by numerical simulations) that a walk of length 1 should be enough to produce a valid preferential attachment, we have found problems with some networks, where there is still a significant correlation between the neighbors average degree and how frequently a certain vertex is marked by a 1-step walk (in pure preferential attachment, the vertex selection criterion is not biased by the vertex neighbors degrees).

\section{Simulation Results}

A number of simulations have been carried out to check the performance of the proposed model along its two main goals: generating a power-law in the degree distribution and controlling the clustering coefficien $t^{5}$

First of all, random walk models require the construction of an initial seed connected network with $n_{0}$ vertices; it is important to point out the influence of the topology of such initial network on the final outcome. As it also happens in the BA model, a non-zero probability must be assigned to the initial isolated vertices (in most implementations this is done by setting a certain parameter $a$ in the distribution so that $p(k) \sim k+a$ ). In order not to bias the first walk, a regular lattice must be chosen where the degree $k_{0}$ is equal for all $n_{0}$ nodes (this is equivalent to the mentioned $a$ parameter in the BA model). Besides, even if we start with a network accomplishing those requirements, the performance

\footnotetext{
${ }^{4}$ The reason why any chosen vertex is first marked instead of directly linked to the new vertex (in step 3 or 4) is because it is desirable for the network to remain unchanged during the addition of the whole $m$ edges. This procedure is common in many implementations of scale-free networks models such as BA, since the hypothesis of the network remaining unchanged during the vertex addition process is used in the mean-field equations model which supports that preferential attachment produces power-laws in degree distribution [22]. In random walk based models, this unchanged network hypothesis is even more important, since the addition of an edge to the last visited vertex can severely change the trajectory of the following random walk.

${ }^{5}$ In our simulations $c_{i}=\left\{\begin{array}{ll}\frac{2 e_{i j}}{k_{i}\left(k_{i}-1\right)} & k_{i}>1 \\ 0 & k_{i} \leq 1\end{array}\right.$, and $C=\frac{1}{N} \sum_{i} c_{i}$. There is also a different clustering degree definition for the global graph in [4] but
} it is not used in this paper. 


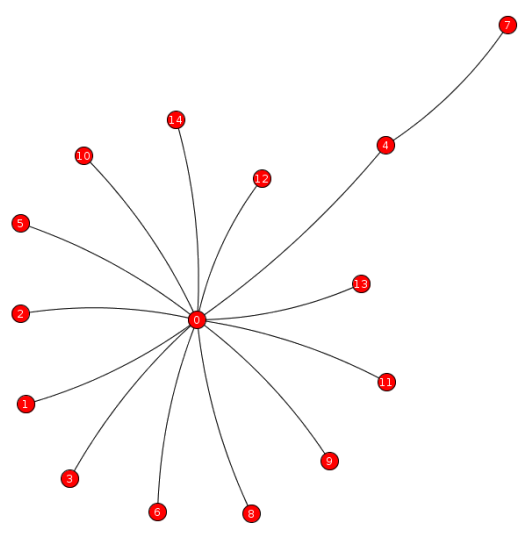

Figure 2. An inappropriate election of $n_{0}$ may drive to a "winner-takes-all" effect. In this simulation $m=1$ and $n_{0}=2$, produce a star-like graph where a power-law degree distribution cannot emerge by a growing process based on preferential attachment.

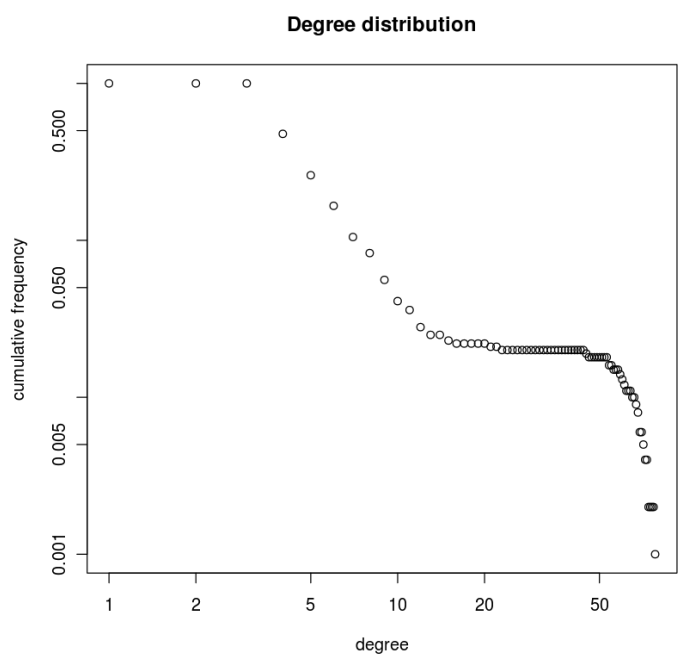

Figure 3. Simulation with $N=10^{3}, m=2$, using a full connected graph of $n_{0}=20$ nodes as a seed. It clearly produces a "step-like" deviation from a power-law tail in the cumulative degree distribution.

of the model might be affected by the values selected for $n_{0}$ and $k_{0}$. Precisely, if $n_{0}$ is too small, there is a high probability of a so called "winner-takes-all" phenomenon to happen as it can be seen in Figure 2. On the other hand, choosing a large value for $k_{0}$ might also create some undesired effects. In this case, choosing a fully connected graph (i.e $\left.k_{0}=n_{0}-1\right)$ might produce a deviation from the power-law behavior in the degree distribution as can be seen in Figure 3. To overcome this situation, a ring graph has been designed, with $n_{0}=\max (10, m)$, which seems to behave properly in most of the stages; hence, all the simulation results presented below make use of this seed. In addition, as pointed out in the model description, a length $l=7$ for the first walk per added node has been used, in order to avoid the dependence on the neighbors degree during the selection process.

\section{A. Scale-free emergence}

Once a proper selection procedure of the initial seed has been settled, we now focus on the first design criterion: the

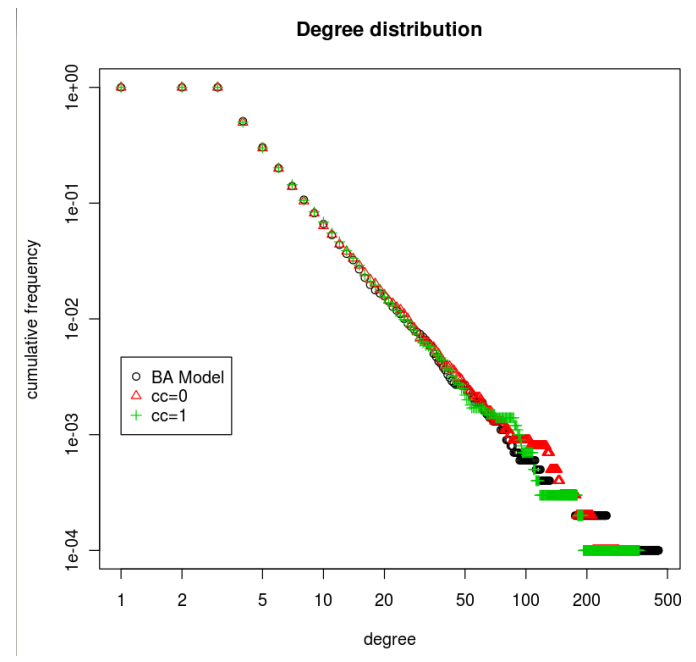

Figure 4. A power-law is obtained in the degree distribution, very close to BA pure preferential attachment model. These results persist under any change in the clustering control parameter $c c$.

emergence of a scale-free network during the growing process. In Figure 4, it can be seen that a power-law is produced (original BA mode ${ }^{6}$ simulation is also included, in order to allow comparison). In addition, it is shown that this behavior does not depend on the value assigned to the clustering control parameter $c c$, as it can be seen in the representation of results for the two extreme values of this parameter. This power-law regime is independent from $c c$ as well as from the size of the network $n$ and the average degree $\langle k\rangle=2 m$; hence, the model proposed here behaves like other preferential attachment based models [2], [10].

\section{B. Clustering coefficient control}

As mentioned earlier, the control of the clustering coefficient is performed by changing the value of the probability $c c$ that a node is assigned " 1 " length value in the binomial distribution characterizing genetic factors. We start by presenting the simulation results which correspond to the first (more intuitive) approach mentioned in Section II, such approach suggested the use of a new random starting vertex for avoiding triangle formation. The results show that this approach drives to a high level of variance for small values of $c c$ as it can be seen in Figure 5. On the other hand, the second approach based on a 2-step mechanism produces a much better performance as shown in Figure 6. Both figures show the clustering coefficient dependence on networks with $N=10^{4}$ nodes and $\langle k\rangle=4$. Mean clustering coefficient (blue points) and standard deviation (red bars), obtained for twenty runs for each value of $c c$, are also presented.

Two additional tests were performed for the proposed model regarding its control capability of the clustering coefficient. The first test proves that the clustering coefficient remains constant for a given $c c$ if the network keeps growing. This result is supported by Figure 7, where the degree is log-plot and the results from $N=1600$ to $N=50 \cdot 10^{3}$ are presented.

${ }^{6}$ For the BA model $a=2$ was selected so that it did agree with our model where a ring (i.e $k_{0}=2$ ) is used as a seed. 


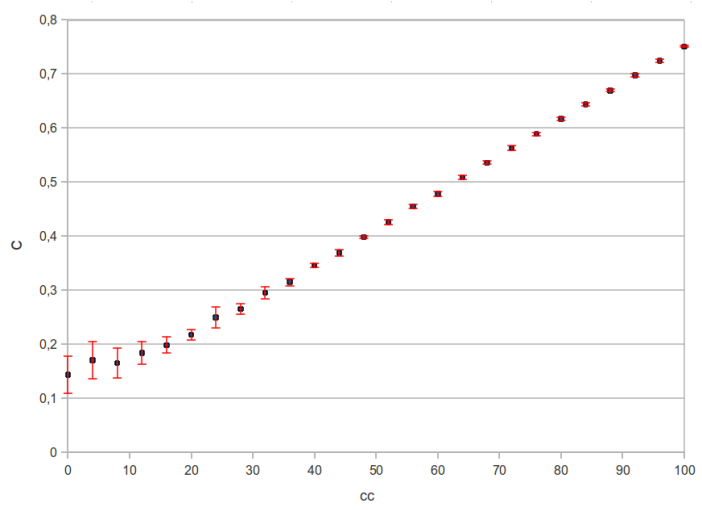

Figure 5. Clustering coefficient control by the new random starting point strategy. The model shows a significant level of variance (red bars represent standard deviation for 20 runs) for small values of $c c$.

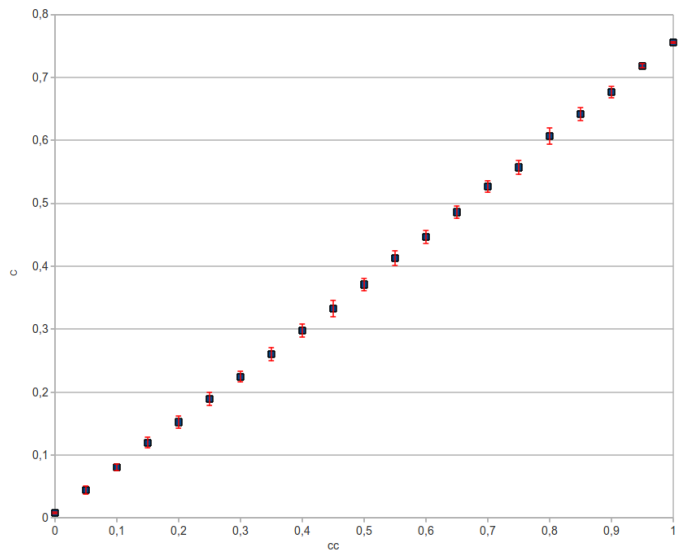

Figure 6. Clustering coefficient control by the 2-step walk strategy. In this case, the model shows a much better performance following a linear relationship characterized by $C=0.74986 \cdot c c$ with $R^{2}=0.99965167$.

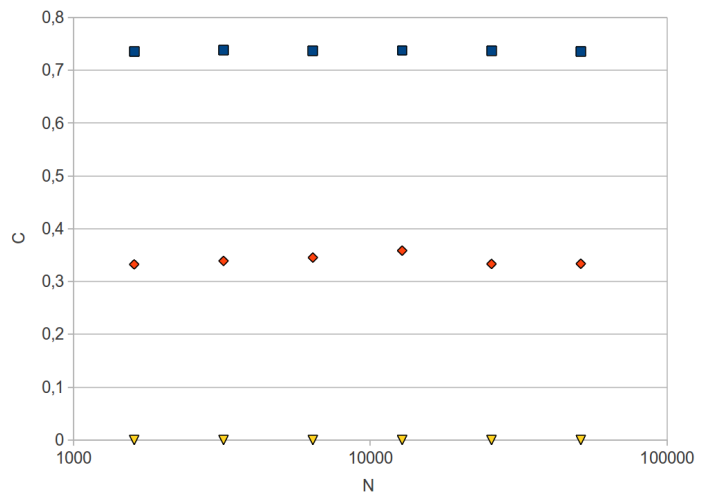

Figure 7. Clustering coefficient variation with network size $N$, for fixed $m=2$. Results are presented for $c c=0$ (yellow), $c c=0.5$ (orange) and $c c=1$ (blue).

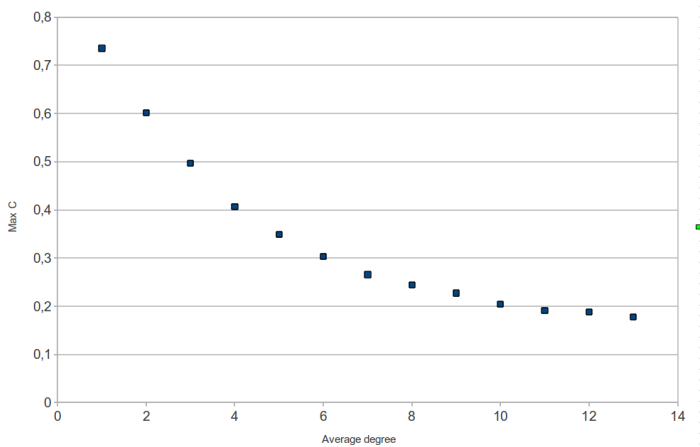

Figure 8. Clustering coefficient decay with average degree.

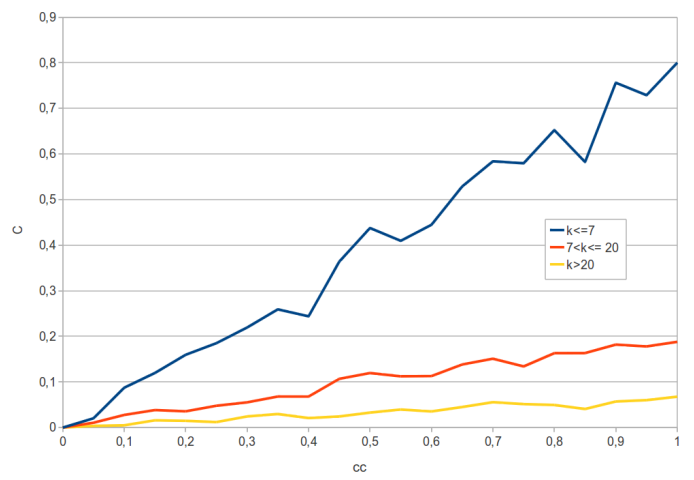

Figure 9. Clustering coefficient control problem for high degree nodes (same simulation parameters as in Figure 6. Nodes are divided into 3 groups according to their degree: the clustering control coefficient $c c$ has smaller influence on the most connected nodes.

The last simulation performed was intended to study the variation of the maximum clustering coefficient that can be generated by the model, that is $c c_{\max }$, as $m$ is increased. The results presented in Figure 8 show a decay of the clustering coefficient as $m$ increases; this expected result shows a behavior equivalent to other tunable clustering network models [13], [28]. In fact, some authors have proposed the use of an alternative clustering coefficient definition since large values of $m$ do bias the degree-clustering correlation in scale-free networks when the standard definition of clustering coefficient is employed. The problem can be summarized as follows: very high-degree nodes (so called "hubs") have very few chances of having a high clustering coefficient; this is due to the fact that it would require most of their neighbors to be connected among themselves, producing a full graph around the hub, which does not fit in a scale-free structure. To support this intuition, a simulation has been performed, whose results are presented in Figure 9, showing that the hubs do not reach high values of clustering coefficient. A new formulation for the clustering coefficient to avoid this degree bias has been proposed in [30]. The performance of our model on this new definition has not been analysed yet.

\section{CONCLUSIONS AND FUTURE WORK}

In this paper, a new scheme for generating scale-free networks with power law degree distribution and tunable clustering coefficient has been presented. The scheme is grounded 
on a combination of random walk and triangle generation procedures together with a genetic factor implementation. These elements allow for an accurate tuning of the clustering coefficient, making use of local information exclusively. As a consequence, this proposed scheme seems to explain the generation of real networks in a more realistic manner. The presented simulations support the validity of the scheme, characterizing its tuning capabilities.

Further research is being carried out in several directions. On the one hand, the sensitivity of preferential attachment policies to the random walk length $l$ is being analysed. On the other hand, some work is also being developed in reproducing additional network metrics by using different $f(p)$ distributions for vertex characterization. An appropriate selection of $f(p)$ can potentially drive to a network where not only average clustering coefficient is controlled, but also the whole clustering coefficient distribution over the network. The distribution $f(p)$ could also be made to depend on some other network metrics (e.g., the degree, so that $f(p, k)$ ) in order to reproduce some correlations between network metrics observed in real networks. Finally, it is worth mentioning that generalizations of this model, based on a network growth driven exclusively by local interaction and intrinsic network attributes, can be implemented in different ways. For instance, some variants proposed in previous random walk models [10] can be easily incorporated to the network model presented here.

\section{ACKNOWLEDGEMENTS}

The authors want to acknowledge the financial support of Orange Spain, in the framework of Cátedra Orange at the ETSI Telecomunicación in the Universidad Politécnica de Madrid (UPM). The work has been also partially supported by projects MTM2010-15102 of Ministerio de Ciencia e Innovación, and Q10 0930-144 of the UPM, Spain.

\section{REFERENCES}

[1] R. Albert and A.L. Barabási. Statistical mechanics of complex networks. Reviews of modern physics, 74(1):47, 2002.

[2] A.L. Barabási and R. Albert. Emergence of scaling in random networks. Science, 286(5439):509, 1999.

[3] A.L. Barabási, H. Jeong, Z. Néda, E. Ravasz, A. Schubert, and T. Vicsek. Evolution of the social network of scientific collaborations. Physica A: Statistical Mechanics and its Applications, 311(3-4):590-614, 2002.

[4] A. Barrat and M. Weigt. On the properties of small-world network models. The European Physical Journal B-Condensed Matter and Complex Systems, 13(3):547-560, 2000.

[5] Q. Chen, H. Chang, R. Govindan, S. Jamin, S.J. Shenker, and W. Willinger. The origin of power-laws in internet topologies revisited. In IEEE INFOCOM, volume 2, pages 608-617. Citeseer, 2002.

[6] J. Davidsen, H. Ebel, and S. Bornholdt. Emergence of a small world from local interactions: Modeling acquaintance networks. Physical Review Letters, 88(12):128701, 2002.

[7] S.N. Dorogovstev and J.F.F. Mendes. Evolution of networks: From biological nets to the Internet and $W W W$. Oxford University Press, USA, 2003.

[8] S.N. Dorogovtsev, J.F.F. Mendes, and A.N. Samukhin. Structure of growing networks with preferential linking. Physical Review Letters, 85(21):4633-4636, 2000.

[9] P. Erdos and A. Rényi. \{On the evolution of random graphs\}. Publ. Math. Inst. Hung. Acad. Sci, 5:17-61, 1960.

[10] TS Evans and JP Saramäki. Scale-free networks from self-organization. Physical Review E, 72(2):26138, 2005.
[11] M. Faloutsos, P. Faloutsos, and C. Faloutsos. On power-law relationships of the internet topology. In Proceedings of the conference on Applications, technologies, architectures, and protocols for computer communication, page 262. ACM, 1999.

[12] J.H. Fowler, C.T. Dawes, and N.A. Christakis. Model of genetic variation in human social networks. Proceedings of the National Academy of Sciences, 106(6):1720, 2009.

[13] P. Holme and B.J. Kim. Growing scale-free networks with tunable clustering. Physical Review E, 65(2):026107, 2002.

[14] H. Jeong, B. Tombor, R. Albert, Z.N. Oltvai, and A.L. Barabási. The large-scale organization of metabolic networks. Nature, 407(6804):651654,2000

[15] J. Jost and M.P. Joy. Evolving networks with distance preferences. Physical Review E, 66(3):036126, 2002.

[16] B. Karrer and MEJ Newman. Random graphs containing arbitrary distributions of subgraphs. Arxiv preprint arXiv:1005.1659, 2010.

[17] P.L. Krapivsky and S. Redner. Organization of growing random networks. Physical Review E, 63(6):066123, 2001.

[18] R. Kumar, P. Raghavan, S. Rajagopalan, D. Sivakumar, A. Tompkins, and E. Upfal. The Web as a graph. In Proceedings of the nineteenth ACM SIGMOD-SIGACT-SIGART symposium on Principles of database systems, pages 1-10. ACM, 2000.

[19] M. Li, L. Gao, Y. Fan, J. Wu, and Z. Di. Emergence of global preferential attachment from local interaction. New Journal of Physics, 12:043029, 2010.

[20] F. Liljeros, C.R. Edling, L.A.N. Amaral, H.E. Stanley, and Y. Åberg. The web of human sexual contacts. Nature, 411(6840):907-908, 2001.

[21] A. Mislove, M. Marcon, K.P. Gummadi, P. Druschel, and B. Bhattacharjee. Measurement and analysis of online social networks. In Proceedings of the 7th ACM SIGCOMM conference on Internet measurement, page 42. ACM, 2007.

[22] M.E.J. Newman. The structure and function of complex networks. SIAM review, 45(2):167-256, 2003.

[23] M.E.J. Newman, S.H. Strogatz, and D.J. Watts. Random graphs with arbitrary degree distributions and their applications. Physical Review E, 64(2):26118, 2001.

[24] J.P. Onnela, J. Saramäki, J. Hyvönen, G. Szabó, D. Lazer, K. Kaski, J. Kertész, and A.L. Barabási. Structure and tie strengths in mobile communication networks. Proceedings of the National Academy of Sciences, 104(18):7332, 2007.

[25] S. Redner. How popular is your paper? An empirical study of the citation distribution. The European Physical Journal B-Condensed Matter and Complex Systems, 4(2):131-134, 1998.

[26] M. Ripeanu, I. Foster, and A. Iamnitchi. Mapping the gnutella network: Properties of large-scale peer-to-peer systems and implications for system design. Arxiv preprint cs/0209028, 2002.

[27] J. Saramäki and K. Kaski. Scale-free networks generated by random walkers. Physica A: Statistical Mechanics and its Applications, 341:8086, 2004.

[28] M.Á. Serrano and M. Boguñá. Tuning clustering in random networks with arbitrary degree distributions. Physical Review E, 72(3):036133, 2005.

[29] D. Smith, C.F. Lee, and N.F. Johnson. Realistic network growth using only local information: From random to scale-free and beyond. Arxiv preprint cond-mat/0608733, 2006.

[30] S.N. Soffer and A. Vázquez. Network clustering coefficient without degree-correlation biases. Physical Review E, 71(5):057101, 2005.

[31] R. Toivonen, J.P. Onnela, J. Saramäki, J. Hyvönen, and K. Kaski. A model for social networks. Physica A: Statistical and Theoretical Physics, 371(2):851-860, 2006

[32] A. Vázquez. Knowing a network by walking on it: emergence of scaling. Arxiv preprint cond-mat/0006132, 2000.

[33] A. Vázquez. Growing network with local rules: Preferential attachment, clustering hierarchy, and degree correlations. Physical Review E, 67(5):056104, 2003.

[34] D.J. Watts and S.H. Strogatz. Collective dynamics of small-world networks. Nature, 393(6684):440-442, 1998.

[35] J.G. White, E. Southgate, JN Thomson, and S. Brenner. The structure of the nervous system of the nematode Caenorhabditis elegans. Philosophical Transactions of the Royal Society of London. B, Biological Sciences, 314(1165):1, 1986. 